Check for updates

Cite this: Chem. Sci., 2018, 9, 2898

Received 12th October 2017

Accepted 24th December 2017

DOI: $10.1039 / \mathrm{c} 7 \mathrm{sc} 04430 \mathrm{e}$

rsc.li/chemical-science

\title{
Absolute and relative facial selectivities in organocatalytic asymmetric chlorocyclization reactions $\uparrow$
}

\author{
Nastaran Salehi Marzijarani, ${ }^{a}$ Roozbeh Yousefi, ${ }^{a}$ Arvind Jaganathan, ${ }^{b}$ \\ Kumar Dilip Ashtekar, ${ }^{a}$ James E. Jackson (D) ${ }^{* a}$ and Babak Borhan (iD) *a
}

\begin{abstract}
Though (DHQD) 2 PHAL-catalyzed chlorocyclizations of 1,1-disubstituted olefins show useful (and in some cases, reversible) asymmetric induction, stereochemically complete descriptions of these alkene additions have remained largely unknown. Herein, based on a combination of NMR, derivative, isotope labeling, and computational studies, we present detailed stereochemical analyses of chlorocyclizations of nucleophile-tethered 1,1-disubstituted styryl systems. The selectivities of the two asymmetric bondforming processes, namely electrophilic chlorine attack and nucleophilic ring closure, are thus mapped out independently. Under the established optimal conditions, four related chlorocyclizations were subjected to this analysis. All showed a strong preference for $\mathrm{Cl}^{+}$delivery from the same face of the alkene. However, depending on reaction conditions and substrate identity (carboxylic acid, amide or carbamate), the internal nucleophiles may close with a strong net preference for either syn or anti addition relative to the $\mathrm{Cl}$ atom. Studies of both uncatalyzed and (DHQD) ${ }_{2} \mathrm{PHAL}$-catalyzed processes place new boundary conditions on the role of the catalyst in these reactions.
\end{abstract}

\section{Introduction}

With the advent of effective asymmetric catalytic methods, halocyclizations (and more broadly, electrophilic haloadditions to alkenes) have now emerged as useful tools for synthesis of chiral targets. ${ }^{1}$ Like the Nobel prize-winning asymmetric epoxidation and dihydroxylation reactions, halocyclization to alkenes had been known for many decades. But despite its obvious desirability and earlier valiant attempts, asymmetric control with significant enantiopreferences remained elusive. Reported in 2010, ${ }^{2}$ efforts from our group (and now many others) then discovered effective reagents, catalysts, and conditions to achieve chlorocyclizations with useful levels of stereocontrol, opening the floodgates of empirical exploration and synthetically valuable discovery, but leaving mechanistic understanding behind.

Broadly, stereocontrol requires the reaction environment to be desymmetrized with a chiral catalyst that activates the reaction while guiding bond formation to prefer one face of the alkene. Many asymmetric halocyclizations of alkenes have now been described using a variety of chiral catalysts. Chiral phosphoric acid catalysts or related analogs were shown to catalyze

${ }^{a}$ Department of Chemistry, Michigan State University, East Lansing, Michigan 48824, USA.E-mail: babak@chemistry.msu.edu; jackson@chemistry.msu.edu ${ }^{b}$ Dow AgroSciences LLC, 9330 Zionsville Road, Indianapolis, IN 46268, USA

$\dagger$ Electronic supplementary information (ESI) available. See DOI: 10.1039/c7sc04430e bromo-amination and -etherification by Shi and coworkers; ${ }^{3}$ bromoetherification by Denmark and Burk; ${ }^{4}$ fluoro/bromo/iodo cyclization of amides by Toste and coworkers; ${ }^{5}$ bromo/iodo etherification by Hennecke and coworkers; ${ }^{1 f, 6}$ and iodolactonization by the Ishihara group. ${ }^{7}$ Fujioka and coworkers disclosed an asymmetric bromolactonization of olefins catalyzed by chiral benzene trimers. ${ }^{8}$ Yeung and coworkers showed that bromoamination and etherification can be catalyzed by $\mathrm{C}_{2}$ symmetric seleno-THF analogs with high enantioselectivity, ${ }^{9}$ whereas asymmetric bromolactonization is achieved by chiral pyrrolidines possessing a thiocarbamate functionality. ${ }^{\mathbf{1 0}}$ More recently the Johnston and Hansen groups reported asymmetric iodolactonizations mediated by chiral PBAM and squaramide based catalysts, respectively. ${ }^{\mathbf{1 1}}$ An elegant report by Jacobsen and coworkers showed iodocyclizations mediated by urea/ thiourea catalysts with high enantioinduction. ${ }^{\mathbf{1 2}}$ New binaphthyl analogs reported by Martin and coworkers proved efficient as catalysts for asymmetric bromolactonization. ${ }^{13}$ Most directly relevant to the present report, several research groups including those of Sun, Yeung, Mukherjee, and Tang demonstrated highly enantioselective intramolecular halofunctionalization reactions catalyzed by monomeric cinchona alkaloid derivatives. ${ }^{2 \boldsymbol{b}, \mathbf{1 4}}$ A number of excellent reviews can be consulted for a complete coverage of the literature in this area. ${ }^{\mathbf{1}}$

Our own and others' studies have uncovered several stereoselective halofunctionalizations based on the organocatalyst (DHQD) ${ }_{2}$ PHAL. ${ }^{2 a, 14 b, 15}$ This $\mathrm{C}_{2}$ symmetric cinchona alkaloid derivative efficiently catalyzes the reaction of substrates bearing 
hydrogen-bond donor groups such as unsaturated amides, carbamates, naphthols and carboxylic acids. In all these asymmetric halocyclization reactions, the olefin undergoes electrophilic attack by a halenium ion $\left(\mathrm{X}^{+}\right)$donor with intramolecular ring closure by a pendant nucleophile. Mechanistically, halofunctionalization of alkenes has been extensively studied since their discovery. The exclusive formation of anti-products from olefin halogenation led Kimball in 1937 to propose a stepwise mechanism, with symmetrically bridged haliranium ions as putative intermediates. ${ }^{16}$ However, the groups of Fahey, Sauers and others provided firm evidence for open $\beta$-halocarbenium ion intermediates in halofunctionalizations of unsymmetrical alkenes, especially those with aryl or other $\pi$-delocalizing substituents. ${ }^{17}$ Furthermore, seminal works by Fahey, Poutsma, Williams, and others concluded that neither the bridged nor the open cationic intermediates are completely compatible with the observed experimental outcomes. ${ }^{18}$ Specifically, reaction rates were accelerated by proximity of the intramolecular nucleophile to the alkene, pointing clearly to a concerted $\operatorname{Ad}_{\mathrm{E}} 3$-type mechanism. ${ }^{17 a, 19}$ Our own recent work in this area has reaffirmed and further illustrated the critical role of the nucleophilic addition partner in activating olefins to abstract the electrophilic halenium ion from its donor reagent. Here, the intrinsic halenium ion affinity ${ }^{20}$ of the alkene pi system is boosted by contact with the nucleophile, favoring $\mathrm{Ad}_{\mathrm{E}} 3$-type concerted halofunctionalization. This nucleophile assisted alkene activation (NAAA) ${ }^{21}$ pathway represents a less familiar but often dominant alternative to the usual textbook scheme of stepwise halofunctionalization wherein initial formation of a bridged halonium ion is followed by its anti opening with a nucleophile.

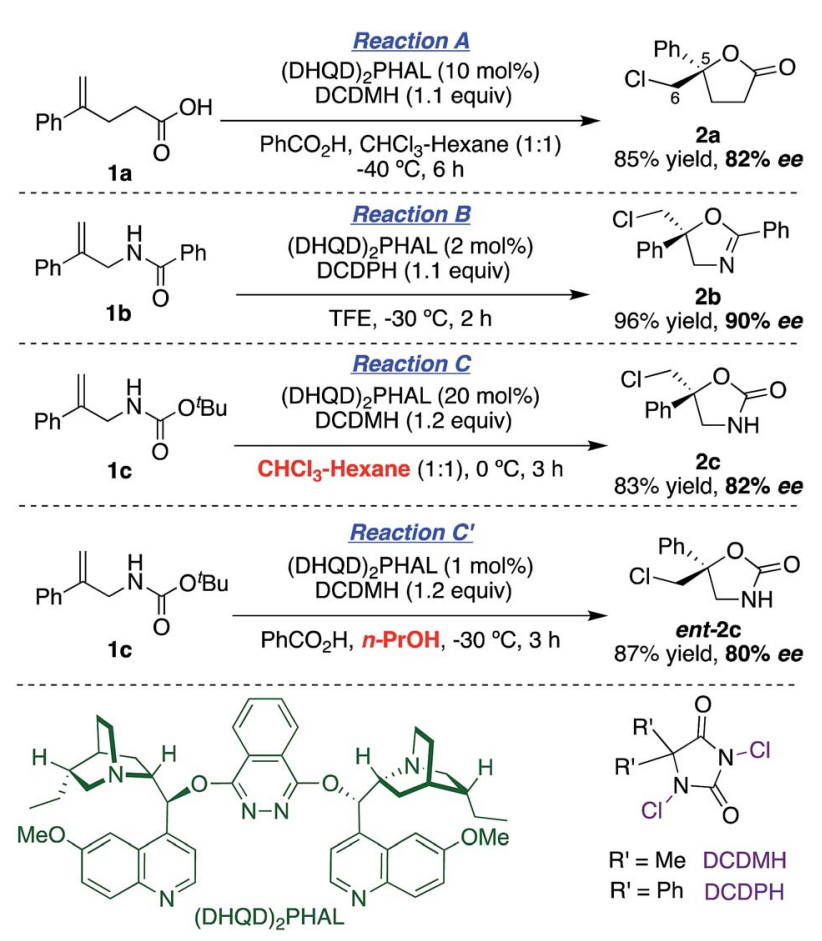

Fig. 1 Asymmetric chlorocyclization of alkenoic acid 1a (Reaction A), unsaturated amide $1 \mathrm{~b}$ (Reaction $\mathrm{B}$ ), and carbamate $1 \mathrm{c}$ under two different conditions (Reactions $C$ and $C^{\prime}$ ).
With asymmetric halocyclizations empirically established as useful synthesis tools, we turned to mechanistic studies of reactions developed in our labs over the past six years. Fig. 1 illustrates the four chlorocyclizations chosen for study. ${ }^{2 a, 15 b, 15 d}$ In the unlabeled systems as originally reported, the newly formed $\mathrm{sp}^{3} \mathrm{CH}_{2} \mathrm{Cl}$ center lacked observable stereochemistry, leaving the face selectivity of chlorination unknown. To reveal the relative and absolute stereochemical outcomes of both the halenium ion attack and the nucleophilic closure in halocyclizations, we resorted to deuterium-labeling in the 1,1-disubstituted olefin substrates. In particular, we investigated the syn: anti selectivity of addition across the double bond, both for non-catalyzed reactions (to evaluate their intrinsic reactivity) and for their (DHQD) $)_{2}$ PHAL-catalyzed analogues.

\section{Results and discussion}

This study sought to uncover and understand the stereochemical relationships between the chlorenium ion delivery and the nucleophilic ring closure events that form the adducts shown in Fig. 1. Some key similarities and differences among the reactions of carboxylic acid 1a, and those of amide $\mathbf{1 b}$ and carbamate 1c, should be noted at the outset. As earlier reported, (a) all these reactions employ (DHQD) ${ }_{2}$ PHAL as the chiral catalyst and chlorinated hydantoins as the electrophilic chlorinating agents. (b) The configuration of the newly created stereogenic carbon (C5) in the product is nucleophile dependent. (c) In the case of the carbamate substrate 1c, the solvent can also modulate the C5 configuration.

To understand these diverse behaviors, we asked the following questions: (a) is there a facial preference for electrophilic chlorine attack on the 1,1-disubstituted olefin? (b) If so, how strong is the preference, and how does it vary among substrates and conditions? (c) What patterns (if any) of stereochemical relationships are found between the chlorine atom and the nucleophile in the final adduct? Net syn or anti halocyclization would shed light on the nature of the reaction path. (d) Is the overall reaction concerted or stepwise; and if the latter, what sort of intermediate (e.g. bridged chloriranium ion, open benzylic carbocation, other?) might be formed? (e) How is the ultimate enantioselectivity set?

As in our previous mechanistic investigations of Reaction $\mathrm{A}^{22}$ the isotopic labeling enables full characterization of the addition stereochemistry in the chlorocyclization of unsaturated amides (Reaction B) and carbamates (Reactions C and $\mathrm{C}^{\prime}$, Fig. 1). The presence of the deuterium in $\mathbf{1 a - D}, \mathbf{1 b}-\mathbf{D}$, and $\mathbf{1 c - D}$ leads to diastereomeric products that reveal not only the face selectivity of chlorenium ion attack on the olefin, but also the syn or anti relationship of addition between the delivered halogen and the captured nucleophile. The results of these studies show a diversity of relative and absolute stereochemical fates in both chlorenium ion delivery and ring closing processes. They also highlight the multiple ways that the catalyst can modulate reactivity.

\section{Synthesis of labeled substrates}

The synthesis of the E-deuterated substrates 1b-D and 1C-D was accomplished in four steps (Scheme 1). The deuterium was 


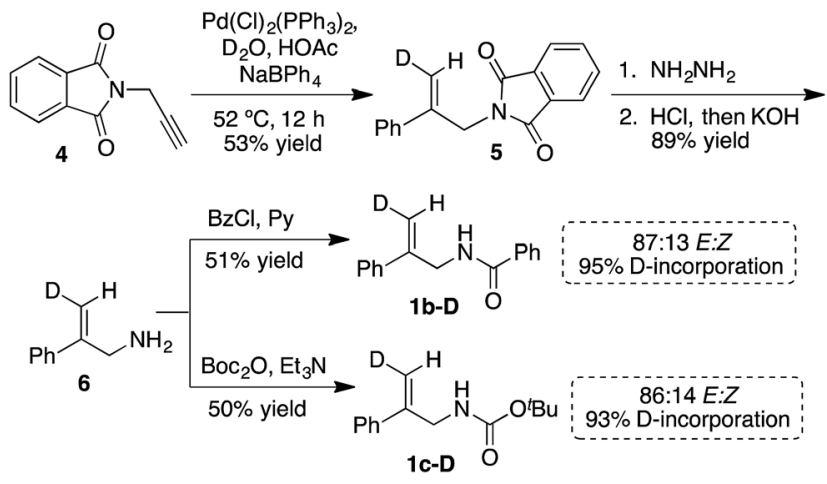

Scheme 1 Synthesis of $1 b-D$ and $1 c-D$.

incorporated through palladium-catalyzed syn hydrophenylation of 4 with sodium tetraphenylborate in $\mathrm{D}_{2} \mathrm{O}$ /acetic acid to afford 5. ${ }^{23}$ Hydrolysis of imide $\mathbf{5}$ and subsequent derivatization of the deuterated alkenoic amine $\mathbf{6}$ with the appropriate acylating agent led to the formation of the desired substrates 1b-D and 1c-D with a high level of deuterium incorporation and good $E / Z$ selectivity. For the final analysis of the chlorocyclization product ratios, the stereochemical impurity ( $\sim 94 \%)$ of the deuterated substrates 1b-D and 1C-D was taken into account by the mathematical treatment shown in the ESI. +

\section{Absolute stereochemical determination}

(DHQD) $)_{2}$ PHAL catalyzed chlorocyclizations of 1b-D and 1c-D (vide infra) led to mixtures of diastereomeric products. The absolute configuration of the deuterated chlorocyclized products 2b-D and 2c-D at C6 was determined via transformation of the major product of both deuterated and non-deuterated substrates to an epoxide with known configuration (Scheme 2, top). Hydrolysis of oxazoline $5 R$-2b-D (absolute configuration at C5 was determined previously by X-ray crystallography ${ }^{15 d}$ with $\mathrm{HCl}$ afforded the $N$-benzoyl $\beta$-amino alcohol 7-D (Scheme 2, top). The resulting halohydrin intermediate was treated with
$\mathrm{K}_{2} \mathrm{CO}_{3}$ to afford the 1,1-disubstituted epoxide 3b-D under mild conditions. Non-deuterated epoxy amide $\mathbf{3 b}$ was synthesized similarly. ROESY and NOESY studies on the epoxy amide $\mathbf{3} \mathbf{b}$ established the relative stereochemistry of $H_{\mathrm{a}}(2.80 \mathrm{ppm}, \mathrm{cis})$ and $H_{\mathrm{b}}(3.10 \mathrm{ppm}$, trans $)$ with respect to the phenyl group. ${ }^{1} \mathrm{H}$ NMR analysis of the epoxy amide $3 \mathbf{b}$-D, obtained from the product of the chlorocyclization of 1 $\mathbf{b}$-D via Reaction B conditions, exhibits the peak at $3.10 \mathrm{ppm}$, establishing that the deuterium has a cis orientation with respect to the phenyl group. This leads to the assignment of $R$ configuration for the carbon bearing the deuterium in epoxy amide $\mathbf{3 b}$-D. Since the epoxy amide is formed through the $\mathrm{S}_{\mathrm{N}} 2$ closure of the corresponding chlorohydrin intermediate, the $S$ configuration at C6 is assigned for amide 2b-D (major product of Reaction B, Scheme 2, top).

In an analogous study, the absolute configuration at $\mathrm{C} 6$ in products obtained from the chlorocyclization of the carbamate 1c-D under conditions denoted as Reaction $\mathrm{C}$ and $\mathrm{C}^{\prime}$ were determined (Scheme 2, bottom). Tosyl protection of oxazolidinone 5R-2c-D and 5S-2c-D (configuration of C5 was established previously via X-ray crystallography), ${ }^{15 b}$ followed by $\mathrm{CsCO}_{3}$ mediated ring opening of the resulting chlorohydrin intermediate, gave 1,1-disubstituted epoxy sulfonamide 3c-D and ent-3cD. The non-deuterated epoxy amide ent-3c was synthesized from $5 S$-2c following the same reaction protocols. ROESY analysis of epoxy amide ent-3c indicated that $H_{\mathrm{a}}(2.75 \mathrm{ppm})$ is cis to the phenyl group, while $H_{\mathrm{b}}(3.20 \mathrm{ppm})$ is trans. ${ }^{1} \mathrm{H}$ NMR data obtained for the epoxides derived from the major products of Reaction $\mathrm{C}$ and $\mathrm{C}^{\prime}$ chlorocyclization reveal that the absolute configuration on the labeled C6 center of the cyclized products $5 R$-2c-D and $5 S$-2c-D is $S$ for both (Scheme 2, bottom).

\section{Stereochemical outcomes of uncatalyzed reactions}

To understand the role of the catalyst in the enantiocontrolled reactions, the intrinsic diastereoselectivities of the uncatalyzed analogues of Reactions $\mathrm{A}-\mathrm{C}$ and $\mathrm{C}^{\prime}$ were first investigated (Fig. 2). ${ }^{24}$ All favor anti addition; in Reaction $\mathrm{A}$,

$$
\text { (R-D }
$$
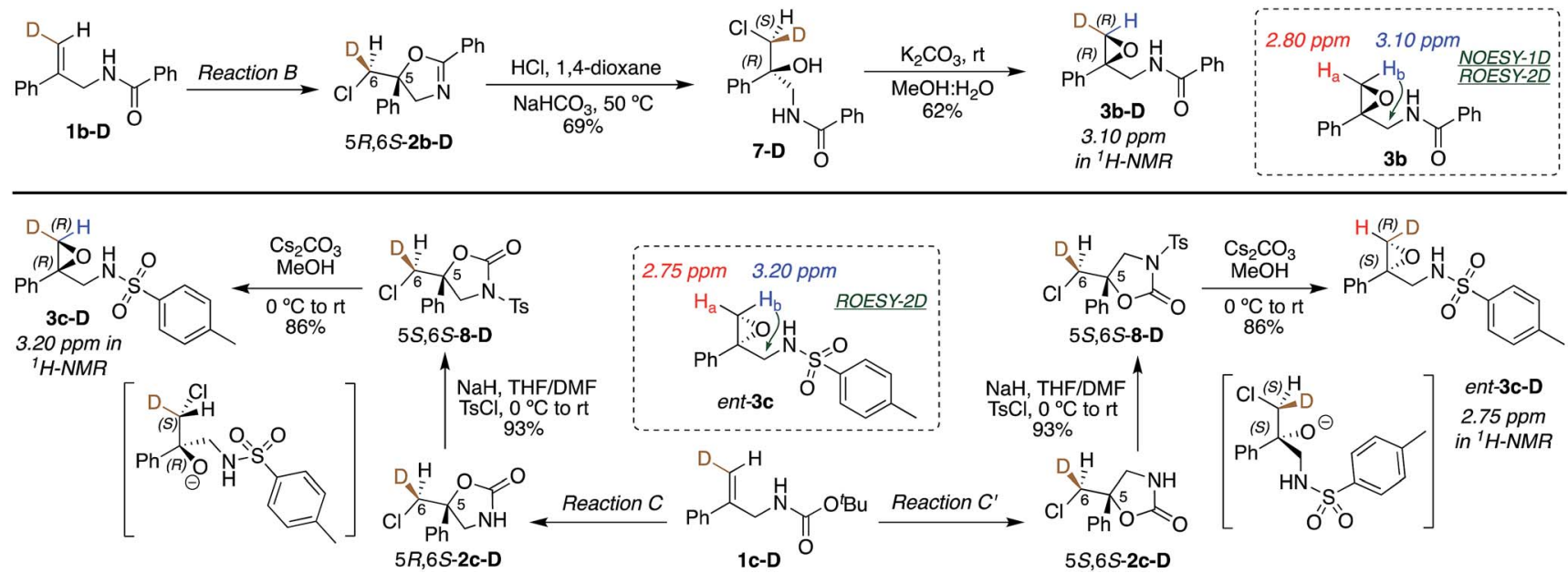

$5 R, 6 S-2 \mathrm{C}-\mathrm{D}$ 1c-D

Scheme 2 Absolute stereochemical assignment at the deuterated center (C6) for substrates 2b-D (top) and 2c-D and ent-2c-D (bottom). 

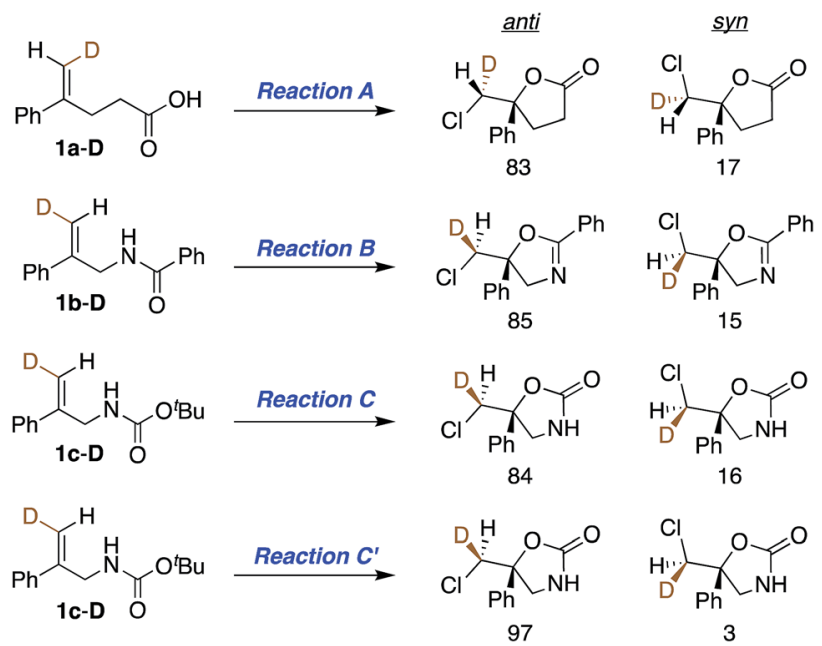

Fig. 2 Summary of intrinsic anti : syn product ratios for uncatalyzed Reactions A, B, C, and C' (enantiomeric pairs are not shown for clarity). Reaction conditions mimic those shown in Fig. 1, except that the catalyst was omitted, and the reactions were run at room temperature.

chlorolactonization occurs with an anti : syn product ratio of $83: 17 .^{22}$ In Reaction B, amidoalkene 1b-D reacts with 1,3-dichloro-5,5-diphenylhydantoin (DCDPH) sluggishly in TFE at room temperature to afford an $85: 15$ mixture of the two diastereomers. Similarly, predominant anti addition is found for the uncatalyzed reaction of carbamate 1c-D with 1,3-dichloro-5,5-dimethylhydantoin (DCDMH), both in $\mathrm{CHCl}_{3}$-hexanes and $n$-PrOH solvent systems; there, anti : syn ratios were 84:16 and 97:3, respectively. Formation of significant quantities of the syn isomer serves as evidence that these reactions do not simply proceed via one path through a stereochemically-defined intermediate (e.g. a cyclic chloriranium ion) able then to dictate stereospecific cyclization to the anti isomer; at least two pathways must contribute to yield the two distinct diastereomers. The substantial shift in anti:syn selectivity (84:16 to $97: 3$ ) between the solvent conditions corresponds to an increase of $\sim 1 \mathrm{kcal} \mathrm{mol}^{-1}$ in the relative barrier for syn $v s$. anti closure; evidently carbamate 1c-D interacts fairly strongly with its surroundings, as expected for such a polar substrate.

To probe the factors that determine anti:syn diastereoselectivity in the absence of catalyst, reaction parameters were systematically studied for chlorocyclization of the amidoalkene 1b-D. First, the effects of the chlorenium ion source were investigated (Table 1, entries 1-8); this factor significantly affects the stereochemical outcome; all yielded mainly anti product, but anti: syn ratios varied from $65: 35$ to $85: 15$. Though the most reactive chlorinating agent (TCCA) does show the lowest selectivity, no direct correlation is seen between chlorenium ion donor ability (assessed via HalA values) ${ }^{20}$ and anti : syn ratios. Thus it appears that the departing moiety of the chlorine donor reagent remains involved at the point of diastereoselection. ${ }^{25}$ What appears unlikely is simple $\mathrm{Cl}^{+}$delivery followed by ring closure in an intermediate free of the leaving group from the chlorinating agent.
The effect of the reaction solvent on anti : syn ratios was next studied (Table 1, entries 9-14). The dr values showed significant solvent dependence; broadly, reaction in solvents of low polarity such as $\mathrm{PhCH}_{3}$ and DCM gave high anti : syn ratios (98:2 and $97: 3)$ whereas relatively polar, hydrogen bonding solvents eroded the anti selectivities. Likewise, running the reaction in a $1: 1$ mixture of $\mathrm{CHCl}_{3}$-hexanes gave a much higher $97: 3 \mathrm{dr}$ than in $\mathrm{CHCl}_{3}$ alone (86:14). These results suggest contributions from mechanisms that are differentially affected by solvent polarity and hydrogen bonding ability. For instance, nonpolar solvents would favor substrate self-association via hydrogen bonds between the amide moieties, ${ }^{26}$ enabling a structurally defined, concerted path to form anti products from reagent + substrate dimers (see Fig. 4a). Polar, hydrogen bonding solvents would disrupt such associations, promoting syn product formation via the more statistically probable $1: 1$ reaction complex (Fig. 4b). We note here that $\mathrm{CHCl}_{3}$ should disrupt substrate aggregation more than $\mathrm{CH}_{2} \mathrm{Cl}_{2} ; \mathrm{CHCl}_{3}$ has long been recognized as the stronger hydrogen bond donor solvent, despite its lower polarity as measured via dielectric constants. ${ }^{27}$ Notably, no intermolecular adducts were seen even in neat solvents capable of serving as nucleophiles $\left(\mathrm{CH}_{3} \mathrm{CN}\right.$ and TFE), or when the amide substrate itself was present in concentrations as high as $0.5 \mathrm{M}$. Thus, any electrophilic intermediates, if formed, must have lifetimes shorter than trapping times in these neat nucleophilic solvents. And even the most aggressive chlorenium ion donors, expected to require little or no nucleophilic assistance, did not trigger intermolecular product formation.

Further mechanistic clues emerged from studies of the effects of reactant concentration on diastereoselectivity. Decreasing the concentration of both the substrate and DCDPH in TFE led to an increase in syn product formation (Table 1, entries 15-20). This is graphically illustrated in Fig. 3, where a steep dropoff in anti: syn ratio occurs at $\sim 0.05$ M. Further explorations varying the individual component concentrations showed little change with varying [DCDPH] but significant falloff in anti selectivity as [1 $\mathbf{1 b}-\mathbf{D}]$ was lowered. These findings support the above suggestion that syn and anti products may arise via mechanisms of different reactant molecularities. More succinctly stated, at high concentrations, an anti-forming transition state could be composed of two (or more) molecules of olefin substrate and one molecule of the DCDPH reactant (Fig. 4a). An obvious mode of interaction involving two substrate molecules would be amide dimerization via $\mathrm{NH}-\mathrm{O}$ hydrogen bonding, altering conformational preferences and enhancing both the effective amide group size and the nucleophilicity of the carbonyl oxygen. Such a complex would be expected to prefer anti addition as depicted in Fig. 4a. Here, the non-reacting amide serves as both the hydrogen bond relay and activator between the DCDPH and the $\mathrm{N}-\mathrm{H}$ site of the reacting amide, enabling chlorenium ion transfer, ring closure, and proton transfer to take place concertedly with minimal charge separation.

In the 1:1 DCDPH : substrate complex that would be entropically favored at lower concentrations, the DCDPH may function as both the base and the chlorenium ion source 
Table 1 Screen of electrophilic chlorinating reagent, solvent and concentration effects on the anti : syn ratios of $2 b-\mathrm{D}$

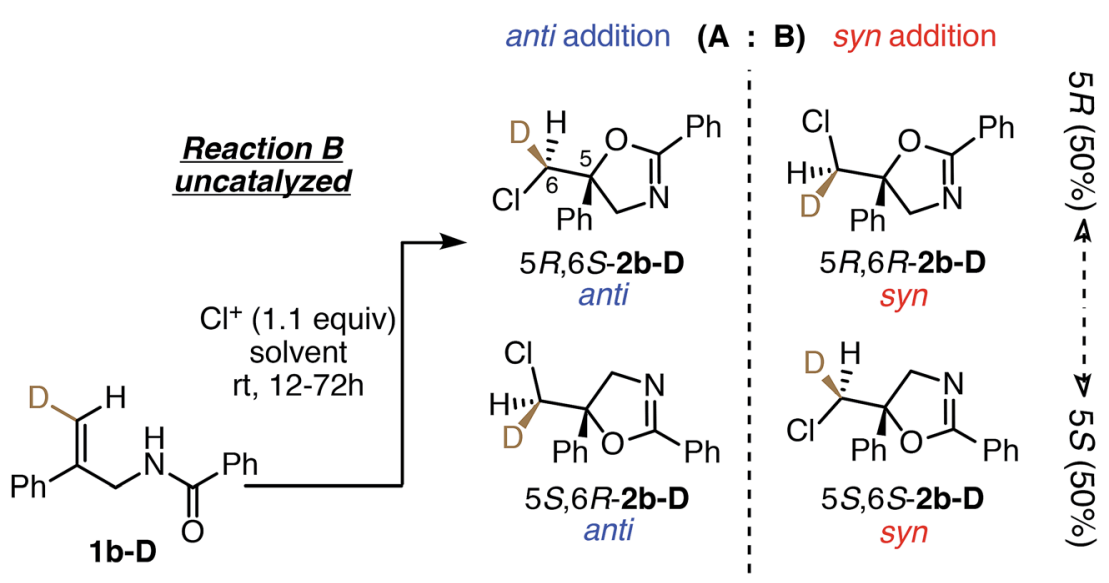

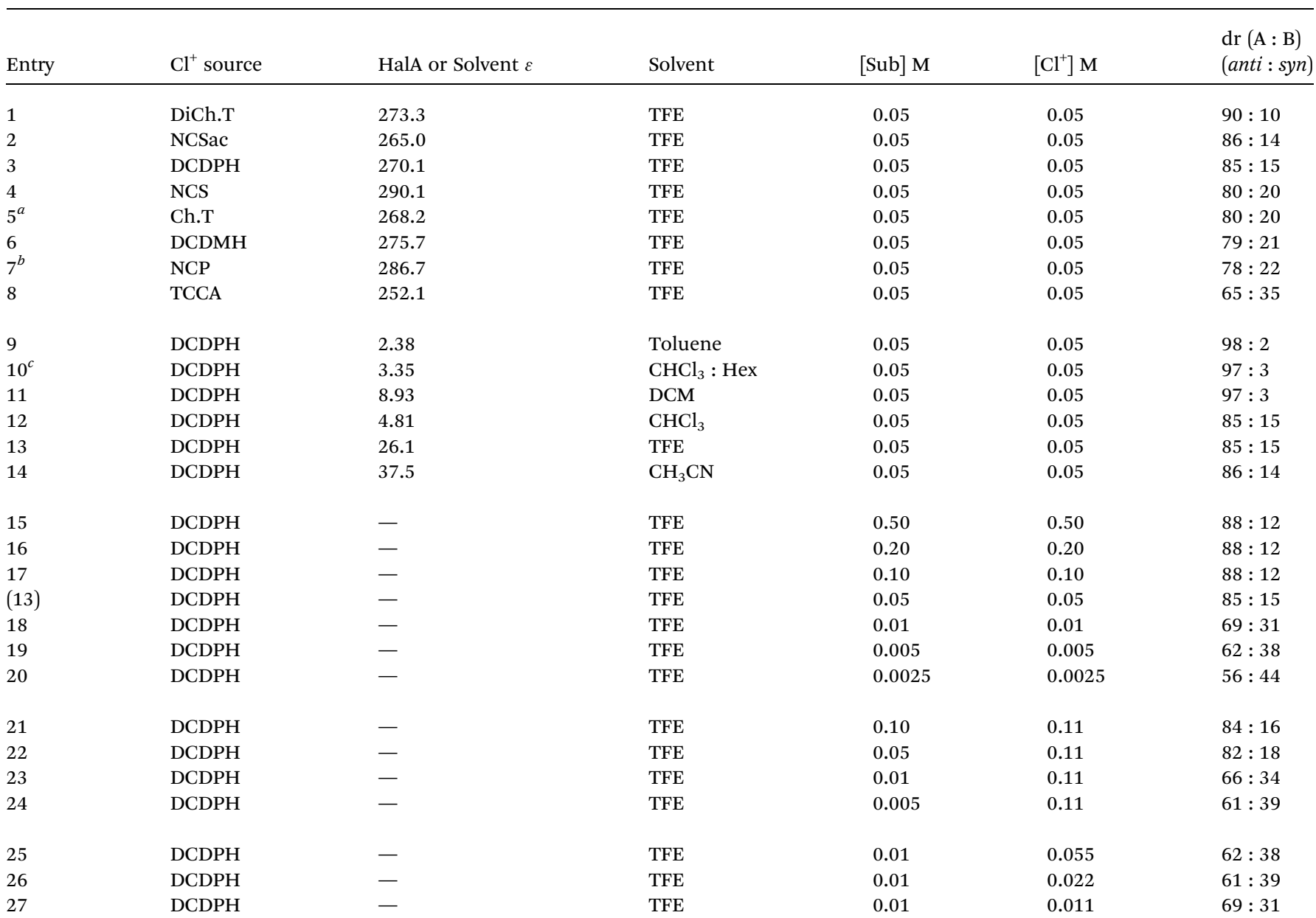

${ }^{a} 58 \%$ conversion after 3 days. $^{b} 82 \%$ conversion after 3 days. ${ }^{c}(1: 1)$ ratio of the solvent; shown dielectric constant is the average of the $\varepsilon$ of the two solvents.

(Fig. 4b), delivering the halogen from the same face as the nucleophile. The amide NH pyramidalization, and the twisting of the alkene seen in the calculated syn transition structure suggests that this species may suffer from substantial strain, consistent with its higher calculated barrier to reaction. On the other hand, the geometries of the hydrogen bonding interactions in the anti TS also appear non-ideal. Nonetheless, these modeled reaction paths are qualitatively consistent with the drop in anti : syn selectivity observed when the concentration of substrate in TFE was decreased from $0.10 \mathrm{M}$ to $0.005 \mathrm{M}$ with [DCDPH] held constant at 0.11 M (Table 1, entries 21-24). Conversely, when the concentration of substrate was kept 


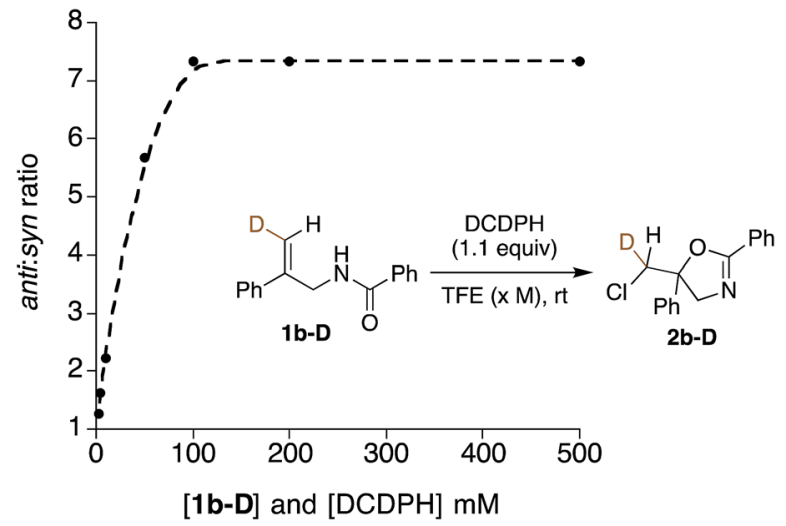

Fig. 3 The plot of anti : syn ratios vs. concentration of DCDPH reagent and $1 \mathrm{~b}-\mathrm{D}$ substrate reaction mixtures.

constant at $0.01 \mathrm{M}$ in TFE and concentration of DCDPH was lowered from $0.11 \mathrm{M}$ (10.0 equiv.) to $0.011 \mathrm{M}$ (1.1 equiv.), no significant changes were observed in diastereoselectivity (Table 1, entries 25-28). These findings point to a scenario with more than one substrate molecule but only one chlorinating agent in the transition state for anti cyclization (Fig. 4a).

Quantum chemical modeling of the above bi- and trimolecular complexes at the T1//EDF2/6-31G* level, with TFE "solvation" simulated with the SMD solvent model, ${ }^{28}$ did indeed find a lower energy path for the termolecular than for the bimolecular process, as seen in Fig. 4; the energetics of these species are summarized in the ESI. $\dagger$ The free energy barriers from separated starting materials via these respective TS structures place the termolecular complex ca. $15 \mathrm{kcal} \mathrm{mol}^{-1}$ lower in energy than the bimolecular case. Solvation in TFE and

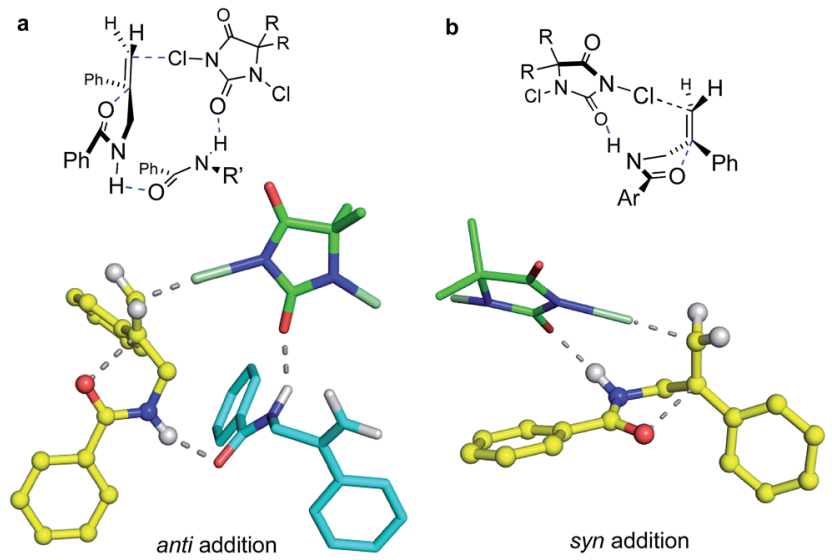

Fig. 4 DFT-computed models for anti (a) and syn (b) cyclo addition of 1b. For computational efficiency, the dimethyl hydantoin was modeled in place of the diphenyl reagent used in the experiments. The higher propensity for anti addition at higher concentrations is consistent with the ca. $15 \mathrm{kcal} \mathrm{mol}^{-1}$ lower activation free energy calculated for the termolecular addition TS (structure a) in which a second amide substrate molecule bridges from the chlorine-donating hydantoin to the $\mathrm{N}-\mathrm{H}$ site of the cyclizing amide moiety. At lower substrate concentration, the more strained bimolecular path (structure b) leading to syn addition is favored. in chloroform, as simulated at the SMD/EDF2/6-31G* level, modulates both paths but does little to change their relative energies. Aggregation via hydrogen bonded interactions, as seen in the termolecular complex, might seem unexpected in a hydroxylic medium such as TFE; however, this solvent is well known to promote polypeptide folding. ${ }^{29}$ On the other hand, entropy would strongly disfavor this higher molecularity structure at the dilute concentrations where syn products emerge.

Lastly, we investigated the effect of altering the electronic properties of the amide aroyl group on the diastereoselectivity of the chloro amidoalkene cyclization (Fig. 5). The largest change is seen with the 3,5-dinitro substituted benzamide 11bD, which yields a near $1: 1$ anti : syn ratio of products 14b-D. Here, we speculate that reduced carbonyl group nucleophilicity in this substrate weakens dimer formation via hydrogen bonding, enhancing the contribution from the syn-favoring $1: 1$ reaction. On the other hand, the increased $\mathrm{N}-\mathrm{H}$ acidity could favor interaction with the DCDMH, activating less selective chlorenium ion delivery reaction.

\section{Diastereoselectivity in catalytic asymmetric reactions}

Having probed the intrinsic diastereoselectivity of uncatalyzed Reaction $\mathrm{B}$, we turned to the stereochemical analysis of the (DHQD) $)_{2}$ PHAL catalyzed asymmetric reactions. As reported earlier, chlorolactonization of alkenoic acid 1a-D (Fig. 1, Reaction A) effects syn-selective carboxylate/chlorenium ion addition with an overall $88: 12$ syn : anti preference (Fig. 6, top). ${ }^{22,30}$ In Reaction $\mathrm{B}$, the (DHQD) ${ }_{2}$ PHAL-catalyzed chlorocyclization of deuterated amide 1b-D yields four stereoisomers under the previously reported catalytic asymmetric conditions (Fig. 6, bottom). ${ }^{15 d}$ The facial selectivity of chlorination, as determined via ${ }^{1} \mathrm{H}$ NMR analysis of the HPLC purified diastereomers (see $\mathrm{ESI} \dagger$ for full details), reveals that the anti product is the major component of the mixture (94\%). Thus, (DHQD) ${ }_{2}$ PHAL controls the facial selectivity of chlorenium ion attack, forming the major epimer with a $99: 1$ preference for the $6 S$ configuration, but only moderate $6 S$ selectivity in forming the minor $(5 S)$ product $(73: 27)$. The nucleophilic closure occurs with high selectivity (93: 7 ratio) favoring the $R$ configuration at C5. As such, the two bond-forming events appear to be independently controlled by the catalyst. Noteworthy is the fact that in Reactions A and B (chlorolactonization and chloroamidocyclization,

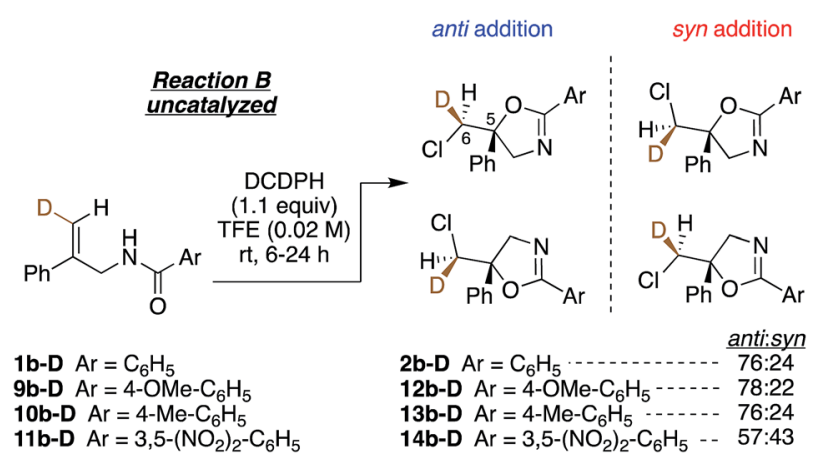

Fig. 5 Anti : syn product ratios of electronically perturbed aryl amides. 

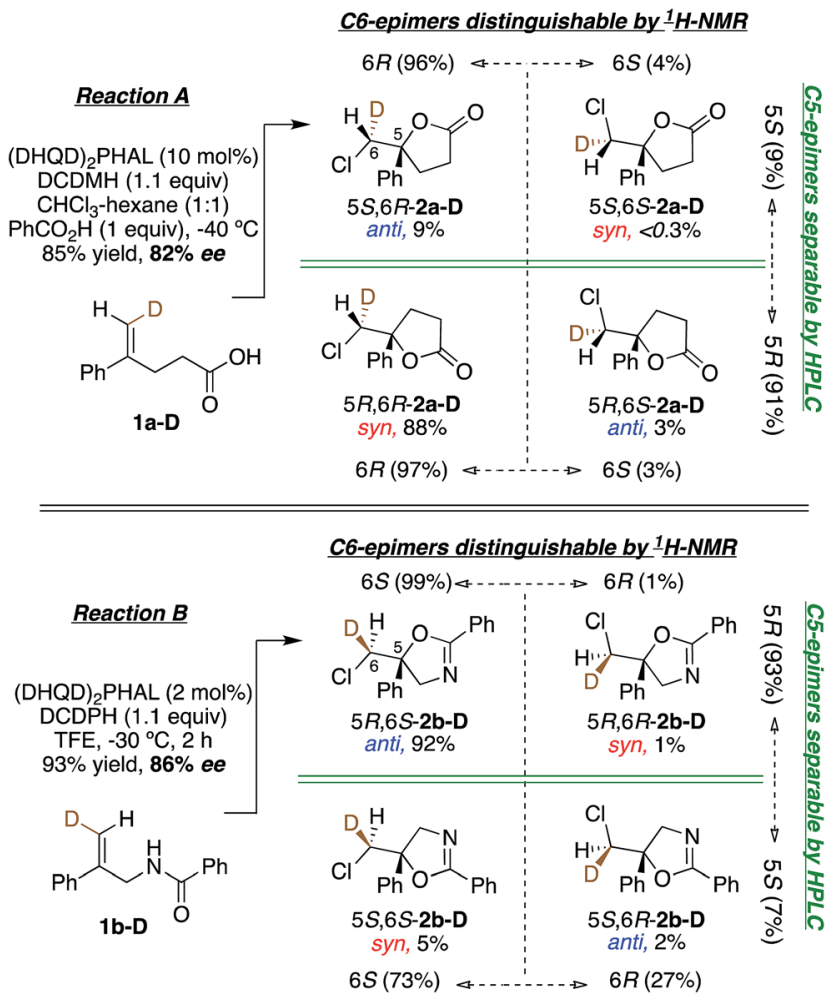

Fig. 6 (DHQD) $)_{2} \mathrm{PHAL}$ catalyzed chlorocyclization of 1-D leads to four isomeric products. Ratios of each isomer are quantified by ${ }^{1} \mathrm{H}$ NMR and HPLC analysis. Reaction A (top, previously reported) shows syn selectivity in net addition of the chlorenium cation and carboxylate anion to olefin 1a-D. ${ }^{22}$ Noting that deuterium differs in configuration between $1 a-D$ and $1 b-D$, and in CIP group priorities about $C 5$ in the product, Reaction $\mathrm{B}$, (bottom) shows predominant anti addition with C6-pro-S/C5-pro- $R$ selectivity in this catalyst templated addition of the chlorine electrophile and the amide nucleophile across the olefin.

respectively), the chlorenium ion delivery occurs to the same face of the olefin, but ring closure occurs on opposite faces of the cyclizing carbon (note that differing substituent priorities designate both products $5 R$, while differing $\mathrm{C} 6$ configurations in 1a-D vs. 1b-D and 1c-D lead to opposite C6 configurations in the respective products 2 for the same facial preference of $\mathrm{Cl}$ attack). The overall process, therefore, is syn for chlorolactonization (Reaction A) and anti for chloroamidocyclization (Reaction B).

In Reactions $\mathrm{C}$ and $\mathrm{C}^{\prime}$, carbamate $\mathbf{1 c}$ shows a switch in the enantiopreference of (DHQD) ${ }_{2}$ PHAL-catalyzed chlorocyclization depending on reaction conditions - primarily the reaction solvent. ${ }^{15 b}$ The overall stereochemistry of both these asymmetric alkene additions is now revealed via deuterated probe 1c-D. Chlorocyclization of 1c-D catalyzed with (DHQD) $)_{2}$ PHAL in $1: 1$ $\mathrm{CHCl}_{3}$ : hexane (Reaction C, Fig. 7, top) yields the anti product $5 R, 6 S-2 \mathrm{c}-\mathrm{D}$ as the major isomer $(85 \%)$; this behavior is thus stereochemically the same as for amide 1b-D. The chlorenium ion delivery to yield products with $6 S$ configuration occurs with high selectivity $(93: 7)$ for the major $(5 R)$ diastereomers. As in the above cases, although the $6 S$ selectivity is still predominant for the minor diastereomer, it occurs with reduced discrimination $(61: 39)$. Thus, the overall face selectivity for
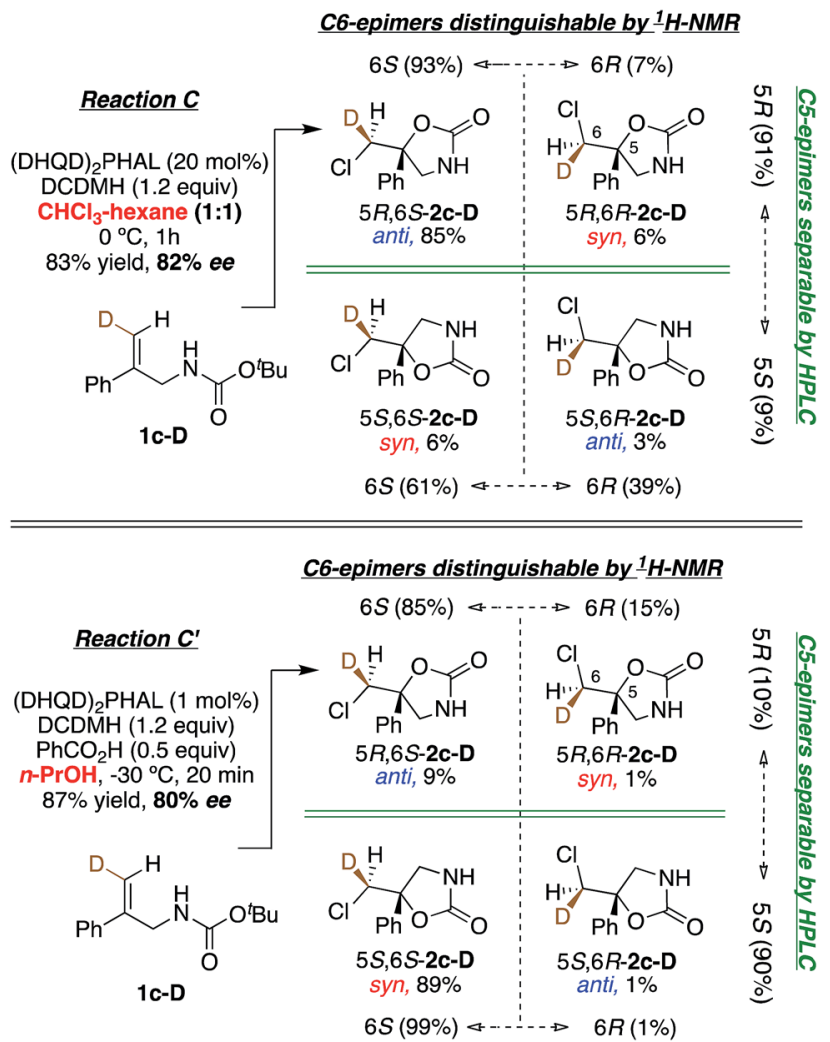

Fig. 7 Chlorocyclization of carbamate $1 C-D$ yields the anti product as the major isomer in $1: 1$ chloroform : hexanes (Reaction C, top), and the syn product as the major isomer in $n-\mathrm{PrOH}$ (Reaction $\mathrm{C}^{\prime}$, bottom). Both reactions are catalyzed by (DHQD) ${ }_{2} \mathrm{PHAL}$.

chlorination on the pro-S face of the $=$ CHD site (i.e. $6 S: 6 R$ ) is 91:9. This C6 stereoselectivity incidentally has the same numerical value as the enantioselectivity of the reaction $(91: 9$ $5 R: 5 S)$.

When applied to Reaction $\mathrm{C}^{\prime}$ (Fig. 7, bottom), the above analysis reveals a contrast relative to both the non-catalyzed reaction in $n-\mathrm{PrOH}$ and the catalyzed Reaction $\mathrm{C}$ in $\mathrm{CHCl}_{3}$ hexanes where anti additions predominate $(97: 3$ and $88: 12$, respectively). Instead, the (DHQD) $)_{2}$ PHAL catalyzed reaction in $n$-PrOH effects syn addition (net anti $:$ syn $=10: 90$ ). However, as in all the other reactions discussed so far, chlorenium ion delivery occurs to the same face, forming the $6 S$ epimers (with this substrate) in high $(99: 1)$ and good $(85: 15)$ enantioselectivity for the major and minor diastereomers, respectively. The net selectivity for chlorination of the C6 pro- $S$ face of $1 \mathrm{c}-\mathrm{D}$ is

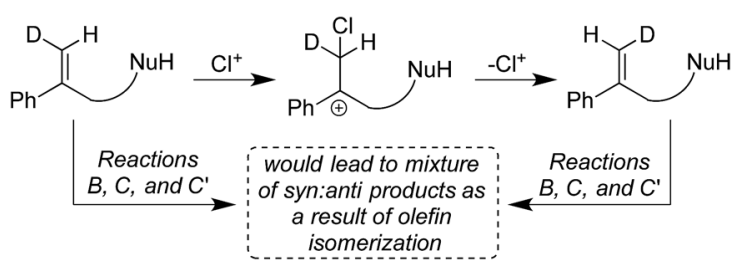

Scheme 3 Possibility of the formation of the isomerized starting material, ruled out by recovery studies, see Table S3. $\dagger$ 
$98: 2$ whereas the C5 face selectivity is somewhat lower, at $90: 10$ pro- $S$.

Given the various syn : anti addition ratios seen as a function of starting material and reaction conditions, it might be suggested that cis-trans isomerization in the starting olefin could explain the observed stereo-randomized products (see Scheme 3 for this hypothetical pathway). This scenario was ruled out (a) implicitly, by the large C6 stereoselectivities seen in some cases (e.g. $99: 1$ in Reaction B), and (b) explicitly, by verification of the stereochemical integrity of labeled substrates 1b-D and 1c-D recovered during the course of Reactions B, C, and $\mathrm{C}^{\prime}$. Reactions quenched at various extents of conversion yield recovered alkenes with $E / Z$ isomeric ratios identical within measurement uncertainties to the starting ratios for all three reactions (see Table $\mathrm{S} 3 \dagger$ ). Besides showing that isomerized reactant is not the source of the disfavored addition products, these results agree with earlier findings that chlorenium ion transfer is not reversible. ${ }^{22,31}$

A summary of the stereochemical outcomes of the various asymmetric chlorocyclization reactions disclosed here is presented in Fig. 8. Most striking is the uniform and selective delivery of chlorine to the same face of C6 in all the alkene substrates. On the other hand, the net syn addition of halogen and nucleophile across the olefin that we had first observed in the initial chlorolactonization chemistry is by no means general. Despite the use of the same organocatalyst and similar hydantoin chlorine sources, different cyclization stereopreferences dominate with different substrates and reaction conditions. Related work from other laboratories ${ }^{5 d, 7,8,10-14,15 a, 32}$ seems likely to exhibit a similar diversity of syn and anti addition across 1,1-disubstituted alkenes.

Though a comprehensive quantum chemical simulation of interacting substrates, organocatalyst, reagents and solvents is beyond the scope of this report, limited simulation studies of the complexation and reaction processes of carbamate substrate 1c were pursued using the Spartan'16 software. ${ }^{33}$ As noted earlier, the ring closure stereochemistry of 1c switches from syn to anti when the medium is changed from $n$-PrOH to $\mathrm{CHCl}_{3} /$ hexane. Based on preliminary NMR studies that suggest a $2: 1$ binding of substrates in the (DHQD) ${ }_{2} \mathrm{PHAL}$ organocatalyst, broad conformational searches on such $2: 1$ complexes were performed with the MMFF94 (ref. 34) and Sybyl $^{35}$ force fields. These simulations typically explored 1-2000

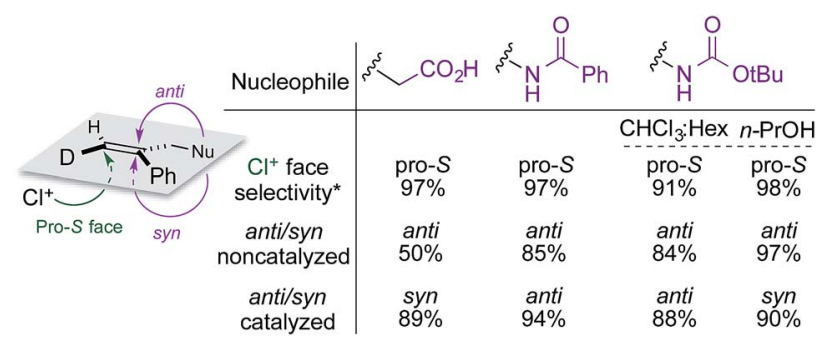

Fig. 8 Summary of stereochemical outcomes for Reactions A, B, C, and $C^{\prime}$. *Configuration designators are listed as shown in the image at left. conformations and were repeated multiple times from various arbitrary starting geometries. They consistently turned up low energy structures that exposed the pro- $R$ alkene face, the experimentally preferred site of chlorine attack. Conformations in the lowest $10 \mathrm{kcal} \mathrm{mol}^{-1}$ range were then re-optimized using the PM6 semiempirical molecular orbital model. ${ }^{36}$ Again, the lowest energy conformations found were orientations with the pro- $R$ face of the alkene exposed. A second series of conformational searches of similar breadth was generated by placing the PM6 calculated transition structures (TSs) for anti and syn chlorocyclizations into one (DHQD) ${ }_{2}$ PHAL pocket, together with a partner non-reacting substrate complexed to the other face. In these cases, the lengths of the partial (reacting) bonds $(\mathrm{N}-\mathrm{Cl}$, $\mathrm{Cl}-\mathrm{C}$, and $\mathrm{C}-\mathrm{O}$ ), were held constant at the gas-phase TS values, but all other degrees of freedom were allowed to vary in the conformational search. Again, the lowest energy set of resulting conformations was reoptimized (still constrained) using PM6, after which full TS optimizations were completed. The resulting syn and anti TSs (each with a single imaginary vibrational frequency corresponding to the alkene addition trajectory) were reevaluated via single-point B3LYP-D3/6-31G* energy calculations, ${ }^{28 a, 37}$ with solvation corrections for chloroform and $n$ PrOH solvents (dielectric constants of 4.8 and 20.3) computed via the C-PCM method. ${ }^{38}$

The final complexes identified via the above procedures are displayed in Fig. 9. Given the relatively low level of theoretical models used to develop them, these structures must be understood mainly as proposed guides for visualization. Nonetheless, we were encouraged by the predominance of low energy catalyst-substrate complex conformations (Fig. 9a) that orient the alkene to expose the face that is actually chlorinated. Likewise, the close energies calculated for the anti and syn transition state structures correspond well with the fact that the difference between $\mathrm{CHCl}_{3}$ and $n$-PrOH media can switch the anti/syn addition selectivities of Reactions $\mathrm{C} / \mathrm{C}^{\prime}$. Notably, the relaxed precursor complex (Fig. 9b) positions the carbonyl oxygen close to the alkene plane, suggesting that its rotations to approach to either side might face similar barriers. Whether closing in anti or syn modes (Fig. 9c and d, respectively), the extended carbamate backbone lying in the catalyst groove must fold up to a TS conformation that brings the carbonyl into contact with the alkene, activating the chlorine transfer and closing the ring. Both TS structures have thus lost factors that stabilized the bound GS, specifically van der Waals interactions between substrate $t$-butyl groups and the phthalazine ring, and among substrate phenyl groups and the quinoline side "walls" of the catalyst. The amide $\mathrm{N}-\mathrm{H}$ site, however, remains associated with catalyst nitrogen atoms by hydrogen bonding, which also activates the nucleophilicity of the carbonyl oxygen as it closes to form the oxazolinone ring.

Energetically, the TS structures for anti and syn (Fig. 9c and d) are calculated to be almost $40 \mathrm{kcal}^{\mathrm{mol}}{ }^{-1}$ higher than the relaxed (DHQD) ${ }_{2}$ PHAL-carbamate-DCDMH complex (Fig. 9b) at the B3LYP-D3/6-31G*//PM6 level. These high DFT-based barriers change surprisingly little upon extension of the calculations to include CPCM simulated $n$-PrOH and $\mathrm{CHCl}_{3}$ solvation environments. Interestingly, the pure PM6//PM6 barriers fall in 

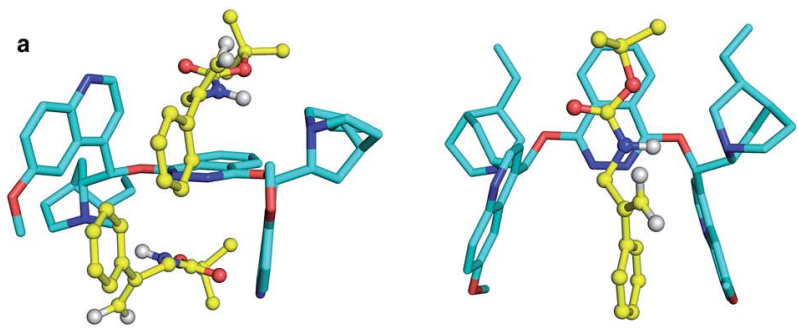

$(\mathrm{DHQD})_{2} \mathrm{PHAL}+1 \mathrm{c}(2$ molecules $)$

b

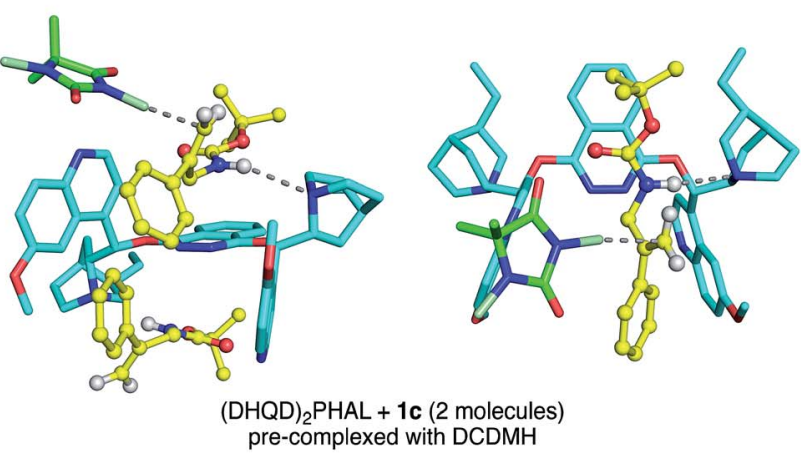

c
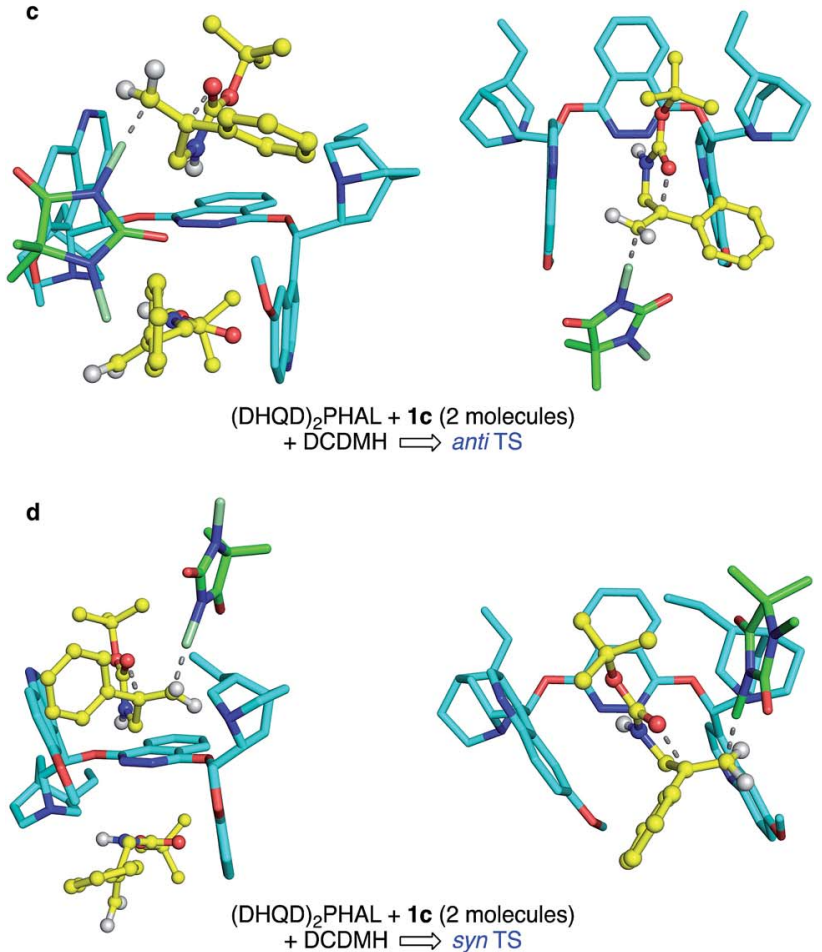

Fig. 9 Side (left) and top (right) view of (DHQD) ${ }_{2} \mathrm{PHAL}$ complexed with $1 \mathrm{c}$ and DCDMH. Hydrogen atoms are not shown in all structures except for the substrate amide and vinylidene protons. In the top view, the second bound substrate is omitted for sake of clarity. (a) (DHQD) ${ }_{2} \mathrm{PHAL}$ bound with 1c. (b) Ground state, pre-complexed interaction of DCDMH with $1 \mathrm{c}$, bound to catalyst, showing the preferred olefin face selectivity as a result of the orientation of the substrate. (c) The anti TS, illustrating a concerted path to product formation. (d) The syn TS, also in a concerted path toward product.

the much more reasonable $20 \mathrm{kcal} \mathrm{mol}^{-1}$ range (see SI for summary) with a small $\left(4.1 \mathrm{kcal} \mathrm{mol}^{-1}\right)$ free energy preference for syn. We suggest that the more intimately bound complexes in Fig. 9a and b are over-stabilized by the well-known overestimation of van der Waals interactions in B3LYP and related DFT methods, augmented by the basis set superposition errors of the relatively small $6-31 \mathrm{G}^{*}$ basis set. Unfortunately, the solvation calculations, which include no geometry relaxation or explicit solvent interactions, offer little insight into the solventswitched selectivities of Reactions $\mathrm{C} / \mathrm{C}^{\prime}$.

The work presented above highlights one of the fundamental challenges in developing highly enantioselective halocyclizations and more generally, additions across alkenes. Here, exceptional face-selectivity in the alkene chlorination is no guarantee of a strong enantiopreference for the newly created C5 $\mathrm{sp}^{3}$ stereocenter; syn and anti addition paths are in close competition. Conversely, a finding of poor enantioselectivity at C5 does not imply poor face-selectivity in the halogen-alkene bond formation.

With the present array of data, our working mechanistic interpretation is that reaction occurs via intramolecular versions of the $\mathrm{Ad}_{\mathrm{E}} 3$ olefin addition. ${ }^{39}$ Here, preorganization of the catalyst and substrate expose the preferred alkene face to the incoming chlorenium ion donor, while still allowing the nucleophilic moiety the conformational flexibility to fold in to contact either face of the alkene. The calculations suggest that the chlorinating agent may form a weak preassociation with the complexed alkene, but the actual bond-forming addition of the halogen to the alkene requires the nucleophile to fold to a conformation that contacts and activates the pi bond for the concerted addition. We recently explored such concerted paths in the substrate framework of Reaction A, where nucleophile-assisted alkene activation (NAAA) promoted non-catalyzed chlorolactonization of 1,1-disubstituted alkenoic acids. That both syn and anti paths can selectively occur among reactions A-C rules out bridged chloronium ions as stereocontrolling intermediates, as expected for these conjugated, 1,1-disubstituted alkenes.

What remains difficult to explain is the observed variability in the ring closure stereochemistry for the four highlighted reactions. As noted above, the complexed alkenes in our calculated structures (see Fig. 9b) place the carbonyl oxygen nearly in the plane of the alkene, establishing no obvious preference for which alkene face would be more easily accessed. Yet amide and carbamate reacting in a non-polar solvent system (Reactions B and $\mathrm{C}^{\prime}$ ) proceed with anti ring closure in contrast to the syn mode seen for carboxylic acids and for carbamates reacting in polar protic solvents. We propose that the amides and carbamates $\mathbf{1 b}$ and 1c (both more nucleophilic and, with only two freely rotating bonds, less conformationally flexible than 1a) most readily fold "inward" to achieve the pro-anti conformation. In contrast, carboxylic acid 1a is less conformationally restricted and potentially capable of hydrogen bond formation, which enables a preference for it to fold outward and activate the alkene from the same face approached by the chlorine-donating hydantoin. Likewise, folding of the polar carbamate moiety of $\mathbf{1 c}$ out into the solvent could be supported by the more polar $n$-PrOH medium, favoring approach from the same face as the chlorenium ion delivery. The carbamate in $n$ PrOH (Reaction $\mathrm{C}^{\prime}$ ) thus behaves much like the carboxylic acid 1a, yielding mainly syn addition product. 


\section{Conclusions}

For a family of chlorocyclizations, both uncatalyzed and mediated by (DHQD) $)_{2} \mathrm{PHAL}$, the relative and absolute stereochemical outcomes have been fully analyzed. In four distinct (DHQD) $2_{2}^{-}$ PHAL-catalyzed processes-chlorocyclizations of carboxylic acid $\mathbf{1 a}$, of amide $\mathbf{1 b}$, and of carbamate $1 \mathbf{c}^{\mathbf{2 a , 1 5 b , 1 5 d}}$ under two sets of conditions - the chlorine attacks the same face of the olefin. This high facial selectivity for chlorenium ion delivery presumably reflects catalyst-mediated pre-organization of the styrene substrate, directing chlorine donor access to only one alkene face. Cyclization by nucleophilic bond closure can show high syn or anti selectivity depending on the nature of the nucleophile and the medium. Thus, the net stereoselectivities at the two new stereocenters appear to be related only in the sense that one of these concerted paths is strongly preferred over others in the optimized reactions. The resulting structural insights place boundary conditions on any mechanistic hypothesis proposed to further refine and generalize this synthetically versatile class of transformations. Detailed kinetic analyses and simulation efforts are ongoing to probe molecularity, catalyst-substrate-reagent binding, preferred conformations in different settings, and reaction rate effects.

\section{Conflicts of interest}

There are no conflicts to declare.

\section{Acknowledgements}

We gratefully acknowledge the NIH (GM110525) and the NSF (CHE-1362812) for funding. The authors thank Dr Daniel Holmes (NMR), and Professor Daniel Jones (mass spec).

\section{Notes and references}

1 (a) A. Castellanos and S. P. Fletcher, Chem.-Eur. J., 2011, 17, 5766; (b) G. F. Chen and S. M. Ma, Angew. Chem., Int. Ed., 2010, 49, 8306; (c) Y. A. Cheng, W. Z. Yu and Y. Y. Yeung, Org. Biomol. Chem., 2014, 12, 2333; (d) S. E. Denmark, W. E. Kuester and M. T. Burk, Angew. Chem., Int. Ed., 2012, 51, 10938; (e) U. Hennecke, Chem.-Asian J., 2012, 7, 456; $(f)$ U. Hennecke and M. Wilking, Synlett, 2014, 25, 1633; $(g)$ C. K. Tan and Y. Y. Yeung, Chem. Commun., 2013, 49, 7985; (h) C. K. Tan, L. Zhou and Y.-Y. Yeung, Synlett, 2011, 2011, 1335; (i) S. Q. Zheng, C. M. Schienebeck, W. Zhang, H. Y. Wang and W. P. Tang, Asian J. Org. Chem., 2014, 3, 366; (j) J. R. Wolstenhulme and V. Gouverneur, Acc. Chem. Res., 2014, 47, 3560; (k) S. R. Chemler and M. T. Bovino, ACS Catal., 2013, 3, 1076; ( $l$ ) J. Chen and L. Zhou, Synthesis, 2014, 46, 586; (m) U. Hennecke, Angew. Chem., Int. Ed. Engl., 2012, 51, 4532; (n) K. Murai and H. Fujioka, Heterocycles, 2013, 87, 763; (o) A. Rouf and S. C. Taneja, Chirality, 2014, 26, 63; (p) S. A. Snyder, D. S. Treitler and A. P. Brucks, Aldrichimica Acta, 2011, 44, 27; (q) C. K. Tan, W. Z. Yu and Y. Y. Yeung, Chirality, 2014, 26, 328.
2 (a) D. C. Whitehead, R. Yousefi, A. Jaganathan and B. Borhan, J. Am. Chem. Soc., 2010, 132, 3298; (b) W. Zhang, S. Zheng, N. Liu, J. B. Werness, I. A. Guzei and W. Tang, J. Am. Chem. Soc., 2010, 132, 3664.

3 (a) D. Huang, X. Liu, L. Li, Y. Cai, W. Liu and Y. Shi, J. Am. Chem. Soc., 2013, 135, 8101; (b) D. Huang, H. Wang, F. Xue, H. Guan, L. Li, X. Peng and Y. Shi, Org. Lett., 2011, 13, 6350. 4 S. E. Denmark and M. T. Burk, Chirality, 2014, 26, 344.

5 (a) K. Hiramatsu, T. Honjo, V. Rauniyar and F. D. Toste, ACS Catal., 2015, 6, 151; (b) V. Rauniyar, A. D. Lackner, G. L. Hamilton and F. D. Toste, Science, 2011, 334, 1681; (c) H. P. Shunatona, N. Fruh, Y. M. Wang, V. Rauniyar and F. D. Toste, Angew. Chem., Int. Ed. Engl., 2013, 52, 7724; (d) Y. M. Wang, J. Wu, C. Hoong, V. Rauniyar and F. D. Toste, J. Am. Chem. Soc., 2012, 134, 12928.

6 C. H. Müller, C. Rösner and U. Hennecke, Chem.-Asian J., 2014, 9, 2162.

7 H. Nakatsuji, Y. Sawamura, A. Sakakura and K. Ishihara, Angew. Chem., Int. Ed., 2014, 53, 6974.

8 (a) K. Murai, T. Matsushita, A. Nakamura, S. Fukushima, M. Shimura and H. Fujioka, Angew. Chem., Int. Ed., 2010, 49, 9174; (b) K. Murai, A. Nakamura, T. Matsushita, M. Shimura and H. Fujioka, Chem.-Eur. J., 2012, 18, 8448.

9 (a) F. Chen, C. K. Tan and Y.-Y. Yeung, J. Am. Chem. Soc., 2013, 135, 1232; (b) Z. Ke, C. K. Tan, F. Chen and Y.-Y. Yeung, J. Am. Chem. Soc., 2014, 136, 5627.

10 X. Jiang, C. K. Tan, L. Zhou and Y.-Y. Yeung, Angew. Chem., Int. Ed., 2012, 51, 7771.

11 (a) M. C. Dobish and J. N. Johnston, J. Am. Chem. Soc., 2012, 134, 6068; (b) J. E. Tungen, J. M. J. Nolsoe and T. V. Hansen, Org. Lett., 2012, 14, 5884.

12 G. E. Veitch and E. N. Jacobsen, Angew. Chem., Int. Ed., 2010, 49, 7332.

13 D. H. Paull, C. Fang, J. R. Donald, A. D. Pansick and S. F. Martin, J. Am. Chem. Soc., 2012, 134, 11128.

14 (a) C. B. Tripathi and S. Mukherjee, Angew. Chem., Int. Ed., 2013, 52, 8450; (b) W. Zhang, N. Liu, C. M. Schienebeck, X. Zhou, I. I. Izhar, I. A. Guzei and W. P. Tang, Chem. Sci., 2013, 4, 2652.

15 (a) A. Armstrong, D. C. Braddock, A. X. Jones and S. Clark, Tetrahedron Lett., 2013, 54, 7004; (b) A. Garzan, A. Jaganathan, N. Salehi Marzijarani, R. Yousefi, D. C. Whitehead, J. E. Jackson and B. Borhan, Chem.-Eur. J., 2013, 19, 9015; (c) K. Ikeuchi, S. Ido, S. Yoshimura, T. Asakawa, M. Inai, Y. Hamashima and T. Kan, Org. Lett., 2012, 14, 6016; (d) A. Jaganathan, A. Garzan, D. C. Whitehead, R. J. Staples and B. Borhan, Angew. Chem., Int. Ed., 2011, 50, 2593; (e) L. J. Li, C. X. Su, X. Q. Liu, H. Tian and Y. A. Shi, Org. Lett., 2014, 16, 3728; $(f)$ M. Wilking, C. Muck-Lichtenfeld, C. G. Daniliuc and U. Hennecke, J. Am. Chem. Soc., 2013, 135, 8133; $(g)$ Q. Yin, S. G. Wang, X. W. Liang, D. W. Gao, J. Zheng and S. L. You, Chem. Sci., 2015, 6, 4179; (h) Q. Yin and S. L. You, Org. Lett., 2013, 15, 4266; (i) Q. Yin and S. L. You, Org. Lett., 2014, 16, 2426; (j) A. Jaganathan, R. J. Staples and B. Borhan, J. Am. Chem. Soc., 2013, 135, 14806; $(k)$ B. Soltanzadeh, A. Jaganathan, R. J. Staples and B. Borhan, Angew. Chem., Int. Ed., 2015, 54, 9517; (l) A. Jaganathan and B. Borhan, Org. 
Lett., 2014, 16, 3616; ( $m$ ) R. Yousefi, D. C. Whitehead, J. M. Mueller, R. J. Staples and B. Borhan, Org. Lett., 2011, 13, 608; (n) O. Lozano, G. Blessley, T. Martinez del Campo, A. L. Thompson, G. T. Giuffredi, M. Bettati, M. Walker, R. Borman and V. Gouverneur, Angew. Chem., Int. Ed., 2011, 50, 8105; (o) K. C. Nicolaou, N. L. Simmons, Y. Ying, P. M. Heretsch and J. S. Chen, J. Am. Chem. Soc., 2011, 133, 8134. 16 I. Roberts and G. E. Kimball, J. Am. Chem. Soc., 1937, 59, 947. 17 (a) R. C. Fahey, in Topics in Stereochemistry, ed. E. L. Eliel and N. L. Allinger, John Wiley \& Sons, Inc., New York, NY, 1968, vol. 3, p. 237; (b) R. C. Fahey and C. McPherso, J. Am. Chem. Soc., 1969, 91, 3865; (c) R. C. Fahey and M. W. Monahan, J. Am. Chem. Soc., 1970, 92, 2816; (d) H. Haubenstock and R. R. Sauers, Tetrahedron, 2004, 60, 1191; (e) H. Haubenstock and R. R. Sauers, Tetrahedron, 2005, 61, 8358.

18 (a) R. C. Fahey, J. Am. Chem. Soc., 1966, 88, 4681; (b) R. C. Fahey and C. Schubert, J. Am. Chem. Soc., 1965, 87, 5172; (c) M. L. Poutsma, J. Am. Chem. Soc., 1965, 87, 4285; (d) B. B. Snider and M. I. Johnston, Tetrahedron Lett., 1985, 26, 5497; (e) D. L. H. Williams, E. Bienvenue-Goetz and J. E. Dubois, J. Chem. Soc. B, 1969, 517.

19 Due to the intramolecularity of the process, the typical $\operatorname{Ad}_{\mathrm{E}} 3$ mechanism, with three participating particles in the transition state, is here formally $\operatorname{Ad}_{\mathrm{E}} 2$ (bimolecular), with two components in the transition state. However, common parlance would still refer to it as $\operatorname{Ad}_{\mathrm{E}} 3$, just as closure of a $\beta$-haloalkoxide to epoxide + halide would commonly be described as an $\mathrm{S}_{\mathrm{N}} 2$ process.

20 K. D. Ashtekar, N. S. Marzijarani, A. Jaganathan, D. Holmes, J. E. Jackson and B. Borhan, J. Am. Chem. Soc., 2014, 136, 13355.

21 K. D. Ashtekar, M. Vetticatt, R. Yousefi, J. E. Jackson and B. Borhan, J. Am. Chem. Soc., 2016, 138, 8114.

22 R. Yousefi, K. D. Ashtekar, D. C. Whitehead, J. E. Jackson and B. Borhan, J. Am. Chem. Soc., 2013, 135, 14524.

23 H. Zeng and R. Hua, J. Org. Chem., 2008, 73, 558.

24 The uncatalyzed reactions were carried in a similar fashion as those detailed in Fig. 2 and 3 except at RT and in the absence of (DHQD) $)_{2}$ PHAL. Although the products were obtained as a racemic mixture, the syn:anti ratios show the intrinsic selectivity for each reaction.

25 To explain the observed anti:syn ratios, we envision a concerted $\operatorname{Ad}_{\mathrm{E}}$ 3-like mechanism (formally $\operatorname{Ad}_{\mathrm{E}} 2$ ). ${ }^{19}$ This nucleophile-activated alkene addition (NAAA) scheme ${ }^{21}$ involves concerted bond formation to the alkene by both nucleophile oxygen and chlorine, a mechanism by which the structure of the chlorine donor could modulate selectivity. Presumably, different substrate conformers or aggregates (vide infra) in solution would have variable reactivity with the different chlorinating agents, yielding different proportions of anti vs. syn products.

26 H. L. Schenck and K. W. Hui, J. Chem. Educ., 2011, 88, 1158.

27 M. J. Kamlet and R. W. Taft, J. Chem. Soc., Perkin Trans. 2, $1979,337$.

28 (a) W. J. Hehre, R. Ditchfield and J. A. Pople, J. Chem. Phys., 1972, 56, 2257; (b) C. Y. Lin, M. W. George and P. M. W. Gill, Aust. J. Chem., 2004, 57, 365; (c) A. V. Marenich, C. J. Cramer and D. G. Truhlar, J. Phys. Chem. B, 2009, 113, 6378; (d) W. S. Ohlinger, P. E. Klunzinger, B. J. Deppmeier and W. J. Hehre, J. Phys. Chem. A, 2009, 113, 2165.

29 (a) J. F. Povey, C. M. Smales, S. J. Hassard and M. J. Howard, J. Struct. Biol., 2007, 157, 329; (b) K. Shiraki, K. Nishikawa and Y. Goto, J. Mol. Biol., 1995, 245, 180.

30 A referee has suggested that KIEs due to substrate alkene deuteration might perturb the labelled experiments enough that they are not representative of the parent reactions. The values of asymmetric induction at C5 now show little if any difference between perprotio and monodeuterated cases for Reactions A, C and $\mathrm{C}^{\prime}$, as can be seen by comparing data in Fig. 1 vs. those in Fig. 6 and 7.

31 S. E. Denmark, M. T. Burk and A. J. Hoover, J. Am. Chem. Soc., 2010, 132, 1232.

32 (a) C. S. Brindle, C. S. Yeung and E. N. Jacobsen, Chem. Sci., 2013, 4, 2100; (b) K. Murai, T. Matsushita, A. Nakamura, N. Hyogo, J. Nakajima and H. Fujioka, Org. Lett., 2013, 15, 2526; (c) D. W. Tay, G. Y. C. Leung and Y. Y. Yeung, Angew. Chem., Int. Ed., 2014, 53, 5161; (d) Y. Zhao, X. J. Jiang and Y. Y. Yeung, Angew. Chem., Int. Ed., 2013, 52, 8597; (e) L. Zhou, J. Chen, C. K. Tan and Y. Y. Yeung, J. Am. Chem. Soc., 2011, 133, 9164; (f) L. Zhou, C. K. Tan, X. J. Jiang, F. Chen and Y. Y. Yeung, J. Am. Chem. Soc., 2010, 132, 15474. 33 Spartan'16, Wavefunction, Inc., Irvine, CA.

34 T. A. Halgren, J. Comput. Chem., 1999, 20, 730.

35 T. A. Halgren, J. Comput. Chem., 1999, 20, 720.

36 J. J. P. Stewart, J. Mol. Model., 2007, 13, 1173.

37 (a) A. D. Becke, J. Chem. Phys., 1993, 98, 5648; (b) S. Grimme, J. Antony, S. Ehrlich and H. Krieg, J. Chem. Phys., 2010, 132, 154104; (c) P. c. Harihara and J. A. Pople, Theor. Chim. Acta, 1973, 28, 213; (d) P. J. Stephens, F. J. Devlin, C. F. Chabalowski and M. J. Frisch, J. Phys. Chem., 1994, 98, 11623.

38 V. Barone and M. Cossi, J. Phys. Chem. A, 1998, 102, 1995.

39 An alternative interpretation is that reaction follows a stepwise mechanism via an intermediate (presumably the benzylic carbocation), where catalyst in independent events rigorously controls the facial selectivity of chlorenium ion delivery to the olefin, and then, less precisely, the enantiodetermining cyclization step. The catalyst would then need to be able to consistently guide attacks from the same face of the olefin in every case, but then specifically favor either syn or anti relative closure trajectories of the nucleophile, depending on conditions. As noted in the text, a key strike against this scenario is that no intermolecular cation trapping products (alkenes, ethers or esters) are seen in the product mixtures, even in cases such as Reaction $\mathrm{A}$, where an equivalent of benzoic acid is available, or Reaction $\mathrm{C}^{\prime}$, where the solvent is the alcohol $n$-PrOH. In addition, to the extent that chlorenium ion transfer might be reversible, the absence of isomerized alkenes is further evidence against a cation formed with any significant lifetime. Another concern is the notion of concerted syn addition across an alkene, but actually such events are familiar as in the Diels-Alder and related but more polar cycloadditions. Likewise, pyrolytic carboxylic acid loss from esters is a well-known concerted syn elimination, the microscopic reverse of syn addition. 